\title{
Emisoras musicales juveniles y nuevo consumo musical a través de dispositivos portátiles ${ }^{1}$
}

Óscar Julián Cuesta M. ${ }^{2}$

\begin{abstract}
Resumfen
El artículo presenta los resultados de una investigación que tuvo como objetivo caracterizar las estrategias util lizadas por las emisoras musicales juveniles de Bogotá para mantener 0 aumentar el número de oyentes en el marco de las nuevas lógicas de consumo musical en dispositivos de audio portátiles. Para ello, en una primera etapa se realizaron entrevistas semiestructuradas a los funcionarios de cinco radioestaciones musicales de Frecuencia M odulada, (FM) de la ciudad, con el fin de establecer las estrategias que utilizan. En un segundo momento se realizaron grupos focales con jóvenes de 14 a 17 años y de 21 a 25 años, para determinar que los lleva a sintonizar emisoras musicales cuando tienen acceso permanente a la música que les gusta en dispositivos como mp3 y I pod, entre otros. La investigación estableció que la innovación en contenidos y la interaccion con los oyentes a traves de redes sociales y otros canales virtuales son las principales estrategias para convocar a los oyentes.
\end{abstract}

A su vez, estos sintonizan las emisoras porque presentan novedades informativas y temáticas, abren espacios de intercambio de opiniones vía redes y generan la sensación de cercanía y afinidad con los locutores.

Balabras elave: Música, consumo musical, emisoras juveniles, dispositivos portátiles. 


\title{
ÓsarjuliánCuestaM
}

Emisoras musicales juveniles y nuevo consumo musical a través de dispositivos portátiles,

artículo producto de la investigación

\section{Youth music radio stations and a new pattern of music consumption through mobile devices}

\begin{abstract}
Abstrakt
This article shows research results aimed to characterize the strategies used by youth music radio stations in Bogota in order to maintain or to increase their audience from an emerging notion of musical consumption using audio portable devices. Therefore, in the first stage, semi-structured interviews were conducted among employees to five FM music radio stations in the city, with the aim to establish the strategies used by them. In a second moment, focal groups were developed with teenagers from 14 to 17 years old and young people from 21 to 25 years of age, in order to determine what drove them to listen to such music stations, if they had permanent access to the music they liked to listen to by using devices, like M P3, I pod, and so on. The research showed that innovation in contents and interaction with listeners through social networks and other virtual channels are the stations' main strategies to attract listeners. At the same time, they said they listened to music stations because they show informative and subject novelties, and they also allow to open opinion exchange spaces using networks, and generate a sensation of closeness and affinity with the radio speakers.
\end{abstract}

Key words: M usic, music consumption, youth radio stations, portable devices.

Recibido: 26 de Enero 201

Aceptado: 10 de Mayo de 2011

\section{Introducción}

Desde hace unos lustros se habla de la crisis del mercado discográfico como consecuencia de las nuevas dinámicas digitales que permiten un novedoso consumo musical (Fouce, 2010), que van desde escuchar las canciones en linea en al gun sitio web (como YouT ube) hasta el intercambio de al bumes enteros entre dispositivos portatilles via B luetooth.

Lo cierto es que, ya sea que el oyente descargue o intercambie, la compra de $C D$ es cada vez menor, pues muchas veces los usuarios encuentran la canción de manera gratuita en formatos simples que permiten llevarla en mp3, I pod, teléfonos celulares y memorias USB, entre otros aparatos. 
Revista de

investigaciones UNAD

Volumen 10. Número 1. Junio 2011

Precisamente, el presente artículo da cuenta de dos preguntas. ¿Qué hacen las emisoras musicales juveniles de Bogotá para mantener o aumentar el numero de oyentes cuando, al mismo tiempo, existen dispositivos tecnologicos que permiten escuchar musica segun los deseos individuales? Consecuentemente, ¿que lleva a un ovente joven a sintonizar una emisora musical para escuchar musica, cuando puede tenerla a su gusto en cualquier momento?

La investigación tuvo como primer objetivo determinar las estrategias que tienen las emi soras musicales juvenil es de Bogota para mantener o incrementar su audiencia, dada la competencia que se origina en los nuevos dispositivos tecnologicos que ofrecen archivos musicales al gusto del propietario. En segundo lugar, explicar las razones que llevan a los oyentes jovenes a sintonizar la música ofrecida por las emisoras musicales contemporaneas.

Para entender este propósito es pertinente hacer referencia a al gunos antecedentes conceptuales e investigativos en esta linea. Primero que todo hay que decir que los dispositivos electronicos de almacenamiento y de comunicacion, como los mp3, mp4, celulares de última generacion, I pod, entre otros, permiten que cada usuario almacene y escuche a su gusto la música que desea en cual quier momento y lugar. Esto hace de tal es dispositivos una fuente permanente de entretenimiento, mas aun cuando les permite generar espacios de interaccion, (pueden escuchar e intercambiar canciones mientras chatean) o de mayor diversión (al mismo tiempo escuchar su grupo favorito y jugar en una carrera de autos).

Por su parte, el oyente de una emisora musical no puede programar las canciones que va a escuchar a su antojo puede perder la recepcion de la señal en ciertas zonas y esperar a que se restablezca o cambiar de dial en las franjas de comerciales.

Este fenómeno se hace más interesante en las nuevas generaciones puesto que tienen unas prácticas diferenciales de consumo simbolico y otras formas de interaccion, dada su familiaridad con los recientes dispositivos tecnologicos y las nuevas dinámicas de intercambio comunicacional.

Según se observó en la indagación, no hay gran número de investigaciones que den cuenta de esta dinamica. De hecho, las que más se acercan analizan la relación de las nuevas formas de consumo musical y la crisis de la industria disquera (Fouce, 2010).

Por ell 0 , Fouce (2010) señala que es necesario ampliar las investigaciones sobre la re) ación entre la comunicacion y la musica, pues es un terreno poco indagado. Segun afirma Gonzalez $\left(S_{1} f, p, 10\right)$, "En la actual idad la radio comercial afronta una situacion de incertidumbre en cuanto a su audiencia, por la que tiene que responder en su afan de responder [sic.] a los deseos o gustos de la audiencia y objetivos comerciales" ( $s, c)$. 


\section{ÓsajuliánCuestam}

Emisoras musicales juveniles y nuevo consumo musical a través de dispositivos portátiles,

artículo producto de la investigación

Como se puede observar, existe en la actual idad un reto para las emisoras, pues las nuevas dinamicas de interacción social y de consumo simbólico renovaron las audiencias, cada vez menos especificas y mas dificiles de clasificar.

A unque este fenómeno es amplio y puede ser visto desde diferentes perspectivas, en esta ocasion se quiere observar el fenomeno desde la relacion entre la comunicación y la musica, pues "la música aparece entonces en el epicentro de diversos problemas que sal pican nuestra cultura contemporanea y nuestra comunicacion mediatica: las practicas cotidianas, la construccion de as identidades, las industrias culturales, las tecnologias, los entornos legales, las resistencias politicas y las posiciones éticas" (Fouce, 2010, p. 71).

Dado que la música representa y posibilita diferentes formas de consumo e intercambio de sentido en la culltura contemporánea, es inquietante, por consiguiente, "que la música, ahora paradigma de los cambios generados en el entorno digital, hava tenido tan poca presencia en los estudios de comunicacion" (Fouce, 2010, p. 71).

En este orden de ideas, el presente artículo busca aportar en este campo poco explorado, observando esa relacion de la musica y la comunicacion en las radioemisoras musicales contemporáneas.

En 2008, A quilera, A dell y Sedeño publicaron un trabajo de dos tomos sobre la comunicacion y la musica, donde describian de manera interdisciplinar esta intima relacion, para lo cual observan los vinculos musica-lenguaje, musicamedios y musica-tecnologia. La publicacion de estos autores es el referente mas inmediato para esta investigacion, pues en el nivel nacional no hay trabajos académicos en esta materia.

Por otro lado, el trabajo de Fouce (2010) se convierte en un referenteimportante, dado que el trabaja la crisis del mercado discografico, en relacion con las nuevas prácticas de escucha, lo cual, interesa de manera significativa para esta investigacion. Este autor describe como las nuevas formas de escuchar musica, caracterizadas por las descargas gratuitas y los dispositivos de al macenamiento portatiles, han afectado la industria discografica tradicional.

En palabras de Fouce (2010):

Las formas de consumo musical imperantes, basadas sobre todo en la gratuidad de las redes P2P, aparecen como desafios frente a la industria, incapaz de encontrar un modelo de negocio que permita rentabilizar económicamente el trafico de esas redes, y también para el Estado, que siente que su poder se difumina en Internet y que la necesidad de garantizar las reglas del juego del libre mercado choca con su obligacion de defender los derechos fundamentales como la privacidad de las comunicaciones (p. 66). 
Revista de

investigaciones UNAD

Volumen 10. Número 1. Junio 2011

Dado que se puede asegurar que "la digitalización de la música ha cambiado sin duda las formas en que la gente escucha musica" (Fouce, 2010a, p. 67), es pertinente observar como ese cambio afecta las dinámicas de las emisoras musicales que comercialmente fueron disenadas para que sus oyentes escucharan a sus cantantes, ritmos y grupos preferidos.

Fouce (2010a) asegura que la radio musical hoy sufre un rechazo porque los oyentes jovenes saben que esta manipulada por las industrias musicales, es decir que en las emisoras se privilegian ciertas bandas o cantantes. Algo diferente ocurre con las nuevas dinámicas del consumo musical, donde usted descarga gratuitamente lo que desee y escucha bandas que nunca han sido promocionadas en las grandes estaciones radiales.

La radio musical, que hasta hace poco había sido el aran medio de divulgación de la musica, tambien es fuertemente criticada, sospechosa de anteponer los intereses de la industria a la satisfaccion de sus oyentes. En consonancia con ello surgen con fuerza los sistemas de recomendacion liqados a los grupos (My ypace) 0 a los gustos de los usuarios (L ast.fm). "M yspace mola mucho más que la radio, tú eliges lo que oyes" (Fouce, 2010a, p. 10).

Los trabajos referenciados anteriormente se convierten en los pocos antecedentes para esta investigación, lo que implica un mayor compromiso para ampliar el conocimiento en la materia.

Se han expuestos razones sobre la crisis de la industria musical, en especial por las nuevas dinamicas de consumo e intercambio digital. Sin embargo, no se han desarrollado ampliamente las consecuencias en el ámbito, radial, particularmente en las emisoras musicales juveniles. La investigacion aqui propuesta pretende ampliar el cuerpo teorico en la materia y aportar, de igual manera, en el campo de la musica-comunicacion, también poco explorado.

\section{Metodología}

Se realizaron entrevistas semiestructuradas a directores y funcionarios de programación de cinco emisoras musicales juvenil es de Bogota. Puntualmente, estas fueron las estaciones de radio y las personas entrevistadas: Radioactiva, Andres López; L, os 40 principales, Hector Contreras; La M ega, A lejandro Villalobos;' Radiónica, Alvaro González; y La X, Alejandro M'arín. Estas entrevistas tuvieron como marco el siguiente interrogante: ¿qué hacęn las emisoras musicales juveniles de Bogota para mantener o aumentar el numero de oyentes cuando, al mismo, tiempo, existen dispositivos tecnológicos que permiten escuchar musica según los deseos individuales? 


\section{ÓsajuliánCuestam}

Emisoras musicales juveniles y nuevo consumo musical a través de dispositivos portátiles,

artículo producto de la investigación

En un segundo momento se conformaron grupos focales con jóvenes universitarios de 21 a 25 años y estudiantes de basiça secundaria de 14 a 17 anos, ambos pertenecientes a instituciones de educacion de caracter privado de la ciudad de B ogotat. Los grupos focales se organizaron despues de tener los resultados de las entrevistas, para poder cotejar con los oyentes jovenenes las respuestas de los anteriores y determinar, en consecuencia, si las estrategias de los primeros tenian resultado positivo o, por el contrario, ell os aducian otras razones de sintonia a pesar de poder escuchar su musica preferida en los dispositivos portatiles.

Consecuentemente, la pregunta guía de los grupos focales fue: ¿qué lleva a un oyente joven a sintonizar una emisora musical para escuchar musica cuando puede tenerla a su gusto en cual quier momento?

\section{Resultados y discusión}

La aplicación de las entrevistas permitió observar lo siguiente:

- La mayoría de los entrevistados coinciden en afirmar que el aspecto principal para que un oyente sintonice una emisora musical juvenil y no se quede con el consumo musical que le dan los dispositivos portatil es son los contenidos ofrecidos.

- Los oyentes no sintonizan solamente la emisora porque van a encontrar su canción preferida. Lo hacen debido a que les presentan la canción de manera especial, con introducciones sensibles y con comentarios especializados.

- Álvaro González, de Radiónica, particularmente considera que los nuevos dispositivos revitalizaron la radio, brindan nuevas posibilidades de contenidos e interacción con sus oyentes;', les permiten reinventarse.

- Una posición contraria es la de Andrés López de Radioactiva, quien plantea que su generación es la llamada a apagar la radio en las proximas decadas, es decir que la radio como medio esta condenada por las nuevas dinamicas y tecnologias.

- Héctor Contreras, de Los 40 Principales, asegura que la música que guarda una persona en un dispositivo portátil tiene una intima relacion con la programación de la emisora: el oyente descarga en un Ipod, mp4, etc., la canción que escuchó en la emisora y le gusto.

- La cercanía entre los locutores o presentadores de los programas de las emisoras musicales es fundamental. No se trata solo de poner musica;', es la compenetración que siente el oyente al estar hablando con un amigo de las 


\section{Revista de}

canciones que les agradan (los sentimientos, la risa, el sarcasmo y el chiste, son cualidades que ellos destacan).

- El dispositivo ofrece el archivo musical para escucharlo cuando el oyente desee, pero la emisora musical presenta la informacion sobre el artista, el sencillo, el álbum, la gira de conciertos, etc.

-Alejandro Villalobos, de la Mega, dice que el desplazamiento que logra el dispositivo es poco, pues hoy al gunos de esos aparatos tienen la posibilidad de escuchar emisoras del mundo entero un ejemplo es Wunder radio, software que facilita escuchar emisoras en estos dispositivos).

- González de Radiónica, piensa que una posibilidad importante que encuentran los oyentes en su emisora son los contenidos, no solo musicales sino de otras areas, como cinematográficas, literarias o medioambientales.

- En líneas generales los entrevistados destacan el vínculo entre la emisora, personificada en sus locutores y presentadores, y los oyentes a traves de redes sociales de internet. La participacion en redes es permanente y tiene espacios a lo largo de la programacion.

- El oyente tiene más relevancia en la programación, se escụchan sus dudas y sugerencias, asunto en el que las redes social es de internet juegan un papel de retroalimentacion inmediata y directa.

- Alejandro Marín, de La X, llama a lo anterior "procuraduría musical", que consiste en estar atentos a los gustos y necesidades del oyente, revisar lo que está en auge del pop, ${ }^{3}$ atender los gustos local es, regionales y mundiales.

- Una de las principales estrategias es la consolidación de una correcta dinamica entre la tecnologla y el contenido.

- Otra estrategia es aprovechar cabalmente las páginas web de las emișoras, comenzando por no llevar la radio terrestre a la web, es decir no limitarse a utilizar la pagina para que el oyente pueda escuchar la emisora en linea. Por el contrario, hay que presentar contenidos novedosos, interactivos y multimediales, por ejemplo ver la foto del evento, mirar los videos de la entrevista, etc. Las paginas por lo general difunden las novedades diarias de la emisora como el concurso, la canción de estreno, el invitado especial, entre otras.

- Con respecto al contenido como principal caracțerística que invita a los oyentes a sintonizar emisoras y no escuchar su musica preferida en archivos

3 La $X$ es una emisora especializada en música pop. 


\section{ÓscajuliánđuestaM}

Emisoras musicales juveniles y nuevo consumo musical a través de dispositivos portátiles,

artículo producto de la investigación

almacenados en dispositivos, es pertinente decir que la construcción de estos contenidos procura la innovación en la mayoría de entrevistados.

Por su parte, los grupos focales permitieron identificar lo siguiente:

- En general, las personas escuchan las emisoras por internet o por dispositivos portatiles que permiten sintonizarlas, como memorias mp3 o tel éfonos celulares. Muy pocos lo hacen a través de radio-receptores tradicionales, pues dicen que no les permiten guardar archivos no solo musicales, sino fotos, hojas de texto, etc.

- Las personas aseguran que la razón principal por la que escuchan música en dispositivos portatiles es que guardan las canciones que les gustan y las escuchan cada vez que deseen. Otra rązón a considerar es que estos dispositi,vos no sufren interferencias (no pierden la señal cuando se pasa por un túnel o cuando se sale de la ciudad, por ejemplo).

- Algunos destacaron que con estos, dispositivos pueden hacer varias cosas: guardar archivos, reproducir música, escuchar radio y tomar fotos.

- Hay una reiterada crítica a los largos espacios de publicidad en las emisoras musicales juveniles, franias que quisieran evitar.

- Escuchan radio porque a través de las emisoras musiçales juveniles conocen las canciones nuevas y las actual idades de sus artistas.

- Las personas no dicen que un dispositivo desplace a la emisora sino que se complementan, ya que cada uno brinda cosas especificas para necesidades precisas en momentos puntuales: el dispositivo para la calle, la emisora para la casa, como un ejemplo reiterado.

- A algunos les gusta la emisora porque brinda información, comenta la música, ofrece la oportunidad de escuchar opiniones y no solo pone canciones.

- La programación y los contenidos que ofrece una emisora son un factor que puede explicar la preferencia de algunos por la emisora y no por el dispositivo.

- Con respecto a los contenidos, las personas consideran que hay emisoras monotonas que siempre hablan de los mismos temas, y que hay otras novedosas. La novedad se convierte en un elemento diferenciador para ganar sintonia: en una memoria tienen quinientas canciones y ya saben cuáles son, pero la emisora puede sorprenderlos con algo nuevo. 
Revista de

investigaciones UNAD

Volumen 10. Número 1. Junio 2011

- Algunas personas reconocen que las emisoras atienden y conocen sus gustos y que, en ese sentido, escuchan a los oyentes.

Dentro de la discusión se consideraron diversas circunstancias que, por su actualidad o su validez, es justo mencionar seguidamente.

Se puede observar que existe una directa correlacion entre las estrategias basadas en el contenido, expuestas por los funcionarios de las emisoras, y las razones para escuchar radioestaciones, explicadas por los oyentes de los grupos focales: Esta correlacion esta dada por dos elementos: el contenido y a interaccion emisoraoyente a traves de redes sociales virtuales y otros canales tambien virtuales.

Con respecto al contenido, los funcionarios de las emisoras musicales aseguraron, en lineas generales, que la principal estrategia para cautivar oyentes era la exposicion de contenidos novedosos que tuvieran en cuenta los deseos de los oventes, Estos, por su parte, afirmaron que prefieren a veces la emisora y no el dispositivo portatil porque además de canciones les ofrece contenidos traducidos en información sobre el artista, la cancion, la gira de conciertos o temas que generan humor, opinion o debate.

Las emisoras plantean que procuran tener contacto permanente con sus oyentes a traves de las redes, procurando escuchar sus inquietudes, sugerencias 0 comentarios, que de al guna manera tienen incidencia en la programaçion. A unque no todos los entrevistados afirmaron que las opiniones de los oyentes influian en la programación, sl fue claro que incidian en los contenidos;' esto implica hacer una diferencia entre la programación, vista como una parrilla que organiza programas, y el contenido, como temáticas a desarrollar en los espacios programados.

La mayoría de oyentes de los grupos focales plantearon que un elemento Ilamativo para escuchar emisoras juveniles era la interaccion en las redes sociales. Ellos afirmaron que sentian que los presentadores y locutores de las emisoras los escuchaban y que asi mismo participaban en los contenidos desarrollados por las emisoras. ${ }^{4}$

Lo anterior, según Herrera (2007, p. 178), se da porque los "profesionales cuentan con nuevos mecanismos de interaccion con la audiencia" y, por su parte, "los oyentes disponen de nuevas fórmulas con las que expresar sus particulares puntos de vista, impresiones y opiniones, y con las que, al mismo tiempo, estrechar su contacto con los profesionales".

Es importante decir que esa interacción con, las redes sociales no se limitaba a la relacion emisora-oyentes, sino que en las páginas de internet de las emisoras y a traves de las redes se construye un vincullo oyente-oyente (especialmente en los chats 0 en el intercambio de comentarios en Facebook o Twitter, por ejemplo).

4 "La radio se debe a sus audiencias y son éstas las que se transforman y a medida que lo hacen exigen que la radio también lo haga" (Rodríguez, 2011, p. 7). 


\section{ĆsajuliánCuestaM}

Emisoras musicales juveniles y nuevo consumo musical a través de dispositivos portátiles,

artículo producto de la investigación

El sentido de compañía entre oyente y locutor, característica diferenciadora de la radio, sigue aun vigente. Las personas de los, grupos focales aseguraban que sintonizaban la emisora no solo por escuchar música, porque eso lo podia hacer con el dispositivo portatil, sino por sentir la cercanía que les daba la sensación de estar habl ando de musica y de otros, temas con un grupo de amigos. En esta misma linea los funcionarios entrevistados planteaban que ese acercamiento emocional, manifiesto en el chiste, el sarcasmo, la risa, es fundamental para convocar, al oyente. Por lo general, al igual que el oyente, al locutor de la emisora juvenil le gusta el estilo de música que presenta su estación de radio, desembocando en que el presentador de una emisora de rock es roquero y quienes lo sintonizan tambien lo son.

Al igual que los oyentes, algunos funcionarios entrevistados no ven en los dispositivos portatil es una competencia sino una posibilidad de complementarse. en primer lugar, los oyentes consultados consideran que los nuevos dispositivos tecnologicos les permiten almacenar musica, pero tambien sintonizar su emisora preferida. De hecho los integrantes de los grupos focal es afirmaron que escuchan as emisoras a traves de estos dispositivos ( $\mathrm{mp} 3$, telefonos de uitima generacion, pad, etc.). En segundo lugar, ellos descargan y almacenan en sus dilspositivos las canciones que escucharon en la emisora. En tercer lugar, van y vienen de la emisora al dispositivo y no se quedan exclușivamente en uno: cuando quieren solo musica recurren a uno, y cuando desean información, discusiones temáticas o compañia recurren a otro.

La crítica más reiterada en los grupos focales hacía las emisoras musicales es la excesiva publicidad en ciertos segmentos de los.programas. Por el contrario, en los dispositivos escuchan la musica que ellos mismos programan y las veces que deseen. El asunto de la publicidad es un reto creativo para las emisoras, pues no pueden omitir la pauta publicitaria que es la que les permite funcionar; de lo que se trata es de determinar una forma organizacional que no sea monotona y predecible.

Otro reto importante en este mismo marco de la creatividad es la innovación en contenidos, puesto que los oyentes esperan cosas nuevas y las emisoras juveniles deben estar en esa constante búsqueda, El dialogo oyente-emisora brinda un referente fundamental para la consolidación de contenidos novedosos.

La interacción oyente-emisora permite atender los deseos de los oyentes, - que satisface en cierta medida la incertidumbre planteada por Rodriguez (2011), quien plantea la dificultad de segmentar la identidad de las audiencias en las dinámicas contemporáneas. Mediante la interaccion de las redes sociales y los demás canales virtuales (blog, correo electronico, M SN, chat, encuestas, etc.) las estaciones juveniles pueden tener elementos. para conocer a sus radioescuchas. Precisamente es lo que ocurre con Radionica - asegura Gonzalez en la entrevista realizada - pues su target group está definido por 
Revista de

investigaciones UNAD

Volumen 10. Número 1. Junio 2011

ciertas edades, pero a través de las redes descubren que llegan a muchas otras:

éll lo ejemplifica con una canción de los Rolling Stones, grupo de finales de los años 60 que al mismo tiempo puede tener seguidores nacidos en las décadas del 50,70090 .

A esto se suma la nueva dinámica de la radio multimedia ${ }^{5}$ que permiten las páginas web de las emisoras, donde no solo se escucha el lenguaje radiofonico tradicional, sino se conjuga con la imagen, el texto y el mundo audiovisual. Alejandro Villalobos, de la Mega, habla incluso de la interaccion via webcam, que se da cuando el oyente ve la labor de los locutores en directo.

Una crítica técnica de los oyentes a la radio es que se pierde la recepción de la emisora en al gunos espacios, por ejemplo al entrar a ciertos edificios, ascensores, tuneles 0 al sălir de la ciudad. Ahi es donde el dispositivo que almacena musica tiene la ventaja y se convierte en el preferido.

Esta investigación manejó los grupos focales tratando de marcar dos grupos diferentes, uno de estudiantes de básica secundaria y otro de universitarios, basados en la posibilidad de que este consumo musical y radiofónico fuera distinto, dado que los primeros tienen menos ingresos economicos para adquirir dispositivos de alta tecnología. Sin embargo, no existe mayor diferencia entre los grupos. Uno de los contrastes que se puede destacar es que los universitarios se quejaron por la chabacanería y los contenidos soeces de algunas emisoras. En este caso tambien hay un reto para los creativos de estas estaciones, consistente en construir contenidos que no vayan contra el buen gusto ni hieran la susceptibilidad de los oyentes.

\section{Conclusiones}

La investigación pudo determinar que las estrategias util izadas por las emisoras musicales juvenil les de B ogotá para mantener 0 aumentar el numero de oventes cuando al mismo tiempo existen dispositivos tecnológicos que permiten escuchar musica según los deseos individuales, se centran en dos elementos: la innovacion en contenidos y la interaccion con los oyentes a traves de redes sociales y otros canales virtuales.

I gualmente, los resultados de la indagación permiten determinar quel os oyentes jovenes que fueron consultados siguen sintonizando las emisoras musicales principalmente por estas razones: las novedades musicales, los contenidos

5 En palabras de Rodríguez (2011, p. 7), "lo que ahora hay que rescatar y que representa el verdadero desafío de la convergencia digital es el mercado de consumidores, nuestras audiencias, donde los usuarios de la radio -antes escuchas- tienen la facultad de transformar la información que reciben". 


\section{ĆsajuliánCuestaM}

Emisoras musicales juveniles y nuevo consumo musical a través de dispositivos portátiles,

artículo producto de la investigación

temáticos e informativos, la interacción vía redes y canales virtuales y la identificación con el estilo, lenquaje y humor de los locutores, cualidades que generan cierta simpatía. En pocas palabras, no escuchan la emisora musical solo por escuchar musica.

L os dispositivos portátiles como Ipod, I pad, mp3, no han desplazado a la radio a pesar de que permiten descargar, intercambiar y escuchar musica libremente, ${ }^{6}$ sin la necesidad de esperar que la programen en la emisora. En lugar de competencia, se observa una relacion de complementariedad: el oyente escucha la emisora y descarga las canciones que a traves de ella escucha en su dispositivo; cuando el oyente solo quiere escuchar musica, se queda con este ulltimo; cuando procura compañia e informacion, sintoniza la estacion; cuando esta pierde la senal, recurre a los archivos musicales de aquel.

La radio contemporánea exige una lógica multimedial donde el lenguaje radiofonico esté acompañado de textos escritos, audiovisuales y graficos. Esto es posible, especificamente, en las paginas web de las emisoras, que no pueden ser simplemente un reflejo de la programacion emitida al arre sino todo un medio con unalógica diferencial.

En consecuencia, los profesionales de la radio no pueden limitarse al lenguaje radial sino que dében utilizar múltiples lenguajes que permitann la multimediălidad de la radio. Las, escuelas de comunicacion social, periodismo, locución y produccion radiofónica estan en la obligacion de permanecer atentas a las nuevas dinamicas en este campo.

La construcción de la programación con los oyentes, posibilitada por las redes sociales y los canales virtuales, implica cambiar la tradición vertical de las emisoras del siglo XX, ya que en nuestros días la comunicación es más horizontal y el medio radial ya no impone su mensaje șino que escucha a su oyente y le permite participar en la formulacion de contenidos.

Otro reto para la radio contemporánea lo plantea Álvaro González, de Radiónica: mantener la radio como escenario de la imaginación, pues con la interacción de múltiples lenguajes lalgunos muy arbitrarios como los basados en imágenes explicitas), la radio podria perder esa condicion de alimentar la imaginación de sus oyentes.

Por otro lado, esta investigación sugiere modificar el manejo de la publicidad de manera que haya varios espacios cortos a lo largo de la programación y no segmentos

6 Vale la pena tener presente la siguiente consideración de Aguilera, M., Adell, J. y Borges, E. (2010, p. 37): “En el nuevo escenario comunicativo digital, el intercambio de archivos o el mero cruce de opiniones y valoraciones sobre la música, entre otros fenómenos, ponen también de manifiesto el papel que ésta puede desempeñar en el proceso de personalización del gusto del usuario, así como en la presentación social de su 'yo' más privado pero público a un mismo tiempo. En el acto de compartir u ofrecer música hay, además del evidente propósito de hacer partícipes a los otros de algo placentero y de entrar en relación, también el deseo de difundir el gusto personal y una voluntad de comunicación de la propia identidad". 
Revista de investigaciones UNAD

Volumen 10. Número 1. Junio 2011

largos de varios minutos como ocurre hoy, cuando al aproximarse al final de los treinta minutos o de la hora, todas las emisoras están en segmentos publicitarios. Final mente es necesario decir que las investigaciones sobre las nuevas dinamicas de consumo musical deben continuar, pues es uno de los escenarios que mas ha mutado a partir de las lógicas digitales y las innovaciones tecnologicas de almacenamiento, descarga e intercambio de archivos. I gualmente es pertinente explorar las diferentes maneras como interactuan los nuevos oyentes radiales, val iendose simultaneamente de diversos medios y lenguajes.

\section{Referencias bibliográficas}

Aguilera, M., Adell, J . y Sedeño, A. (Eds.) (2008). Comunicación y música I. Lenguaje y medios. Barcelona: UOC Press.

Aguilera, M., Adell, J ., Borges, E. (2010). Apropiaciones imaginativas de la música en los nuevos escenarios comunicativos. Comunicar, 17(34), 35-44 [en línea]. Disponible en: http://redalyc.uaemex.mx/pdf/158/15812481005.pdf [2011, 8 de junio].

Fouce, H. (2008). No es lo mismo: audiencias activas y públicos masivos en la era de la música digital. En Aguilera, M., Adell, J . \& Sedeño, A. (Eds). Comunicación y música II. Tecnologías y audiencias. Barcelona: UOC Press.

(2009). Prácticas emergentes y nuevas tecnologías. El caso de la música digital en España. Madrid: Fundación Alternativas.

.. (2010a). Tecnologías y medios de comunicación en la música digital: De la crisis del mercado discográfico a las nuevas prácticas de escucha. Comunicar, 17(34), 65-72.

(2010b). Nativos digitales en la selva sonora. Tecnologías y Experiencia cultural en la música digital. Revista Razón y Palabra N. 73(s. p.) [en línea]. Disponible en: http:// www.razonypalabra.org.mx/N/N73/M onotematicoN73/08-M 73Fouce.pdf [2011, 6 de junio].

González, J. (s. f.). Estudio de audiencias a estudiantes de la Pontificia Universidad J averiana en relación a la emisora J averiana Estéreo [en línea]. Disponible en: http:// www.javeriana. edu.co/biblos/tesis/comunicacion/tesis244.pdf [2011, 8 de junio].

Herrera, S. (2007). Las nuevas modalidades para la participación de los jóvenes en la radio española. Revista Trípodos, 20, 171-188.

Rodríguez, E. (2011). Nativos digitales en la reconfiguración radiofónica. Revista Razón y Palabra N. 76(s. p.) [en línea]. Disponible en: http://www.razonypalabra.org.mx/N/ N76/ varia/3a\%entrega/32_Rodriguez_V76.pdf [2011, 6 de junio]. 